\title{
¿Cerrar ahora para reabrir mejor mañana?
}

\author{
La continuidad pedagógica en las universidades \\ de América Latina durante la pandemia*
}

\section{INTRODUCCIÓN}

Tras más de un año de pandemia la mayoría de las universidades e instituciones de educación superior de América Latina continúan cerradas. A medida que las vacunas vayan generalizándose es de esperar que las aulas se reabran, pero por el momento parece difícil establecer una fecha de retorno. En ciertos países, algunas titulaciones permiten ya la vuelta de pequeños grupos de estudiantes para minimizar el impacto de la falta de clases prácticas en laboratorios, de trabajos en taller $y$, sobre todo, de prácticas clínicas. Pero, en gran parte de los casos, la docencia ha entrado de lleno en la llamada educación remota de emergencia, un eufemismo para referirse a la necesidad de utilizar las capacidades disponibles para garantizar la continuidad pedagógica usando fórmulas muy variables de comunicación y de trasmisión de contenidos a distancia. Salvo en aquellos países e instituciones que contaban ya con tradición en materia de educación superior a distancia, en muchos casos no hubo otra solución que improvisar.

Tras una primera fase de emergencia, que se empezó a superar a medida que los gobiernos y las universidades asumían que la duración de la clausura de las aulas iba a proseguir más allá de un curso académico, se ha entrado progresivamente en una fase de maduración apoyada en la generalización o la consolidación de soluciones tecnológicas, así como la cualificación de las estrategias pedagógicas utilizadas (Amemado, 2020). Aunque es todavía pronto para saber cuáles serán los efectos de este proceso de maduración progresiva cuando se reabran las aulas, es inevitable preguntarse acerca de cuáles están siendo los efectos que las soluciones de continuidad pedagógica de base tecnológica empleadas están generando (Goedegebuure y Meek, 2021; Pedró, 2020b).

Hasta el momento se ha producido escasa literatura y aún menos evidencia empírica acerca de la transformación de los procesos de enseñanza y aprendizaje durante la pandemia, no sólo en América Latina sino incluso en otras regiones del mundo más desarrolladas. Hay cuatro notables excepciones: el estudio de UNESCO-IESALC que presenta una panorámica global sobre el impacto de la pandemia en la educación superior (UNESCO-IESALC, 2020), el estudio comparativo de Crawford et al. (2020) que repasa los impactos de la pandemia en la docencia digital en 20 países distintos, la recopilación de evidencias de Farnell et al. (2021), y los resultados de la encuesta de la Asociación Internacional de Universidades (Marinoni et al., 2020), desgraciadamente con una muestra muy reducida de América Latina y el Caribe. En la región parecen más frecuentes los ensayos y las opiniones cualificadas (Gazca Herrera, 2020; Salto, 2020), pero son muy contados los análisis que parten de resultados de encuestas o de entrevistas. Entre ellos hay que destacar el estudio de Camaño

\footnotetext{
* El cierre de instituciones de educación superior por más de 15 meses en América Latina y el Caribe, y en general, en todo el mundo, está teniendo enormes consecuencias en todos los ámbitos que resulta indispensable analizar y ponderar. Por esta razón, hemos considerado importante reproducir en Perfiles Educativos este documento, publicado en 2021 por la Organización de las Naciones Unidas para la Educación, la Ciencia y la Cultura (UNESCO), y por el Instituto Internacional de la UNESCO para la Educación Superior en América Latina y el Caribe (IESALC).
} 
Morúa et al. (2020), que compara las iniciativas en cinco países distintos y 25 universidades, y el de Hershberg et al. (2020), que presenta los resultados de 50 entrevistas a directivos universitarios de la región.

Indudablemente, son un lastre las dificultades inherentes a la pandemia, junto con la falta de tradición de estudios empíricos centrados en la transparencia de los métodos de enseñanza y aprendizaje en la educación superior en la región. Pero, poner de manifiesto cuáles son las estrategias que se están siguiendo, así como los desafíos a los que se enfrentan las universidades, sus docentes y los propios estudiantes para sacar el máximo partido de las soluciones adoptadas, son de interés porque permiten establecer las bases para una discusión ulterior acerca del futuro, calidad y equidad de la enseñanza universitaria en la región.

\section{Objetivos}

El objetivo fundamental de esta investigación consiste en poner de manifiesto las estrategias desarrolladas por las instituciones de educación superior en la región para garantizar la continuidad pedagógica. Se busca, además, comprender el contexto en el que operan las estrategias de continuidad pedagógica, tanto desde un punto de vista tecnológico como pedagógico, así como ofrecer una panorámica de la calidad de los entornos políticos en los que estas iniciativas debieron desarrollarse.

En primer lugar, se indagó sobre las soluciones tecnológicas, digitales o no, que estaban haciendo posible la continuidad pedagógica. En segundo lugar, más allá de la tecnología y su recepción, se buscó arrojar luz acerca de las distintas estrategias pedagógicas propuestas y desarrolladas y el apoyo que las instituciones ofrecían al profesorado para el desarrollo de las necesarias capacidades para optimizar el trabajo docente en contextos de educación remota. En tercer lugar, se pretendió conocer los mecanismos implementados para garantizar el apoyo a los estudiantes, en particular a aquéllos que por razones socioeconómicas o tecnológicas podían encontrarse en situación de mayor vulnerabilidad y, por consiguiente, con riesgo de abandono o de fracaso académico. En cuarto lugar, se quiso profundizar en el entorno político y las condiciones creadas para que las universidades pudieran operar mejor en la fase de educación remota a distancia, conscientes de las dificultades normativas con las que la educación a distancia topa en muchos países de la región. Y, finalmente, se buscó identificar aquellos procesos e iniciativas que podrían ser indicativos de procesos de innovación pedagógica o tecnológica que tuvieran visos de proyectarse hacia el futuro.

\section{Metodología}

Para poder recopilar datos acerca de la continuidad pedagógica en la región se diseñó una encuesta que, fundamentalmente, contenía preguntas acerca de cada uno de los temas señalados. La encuesta fue administrada, a través de la web, a una muestra aleatoria de 100 universidades de América Latina, procesadas entre los meses de abril y junio. La muestra es representativa de la región, pero no para cada uno de los países, por lo que las comparaciones entre ellos deben evitarse o, como mucho, considerarse meramente indicativas. La Tabla 1 presenta la distribución de la muestra por país, abarcando 16 países de la región.

Tabla 1. Composición de

la muestra de universidades encuestadas por países

\begin{tabular}{lc}
\hline \multicolumn{1}{c}{ País } & Porcentaje \\
\hline Argentina & 14 \\
\hline Bolivia & 3 \\
\hline Brasil & 12 \\
\hline Chile & 2 \\
\hline Colombia & 19 \\
\hline Ecuador & 4 \\
\hline El Salvador & 3 \\
\hline Guatemala & 1 \\
\hline
\end{tabular}


Tabla 1. Composición de

la muestra de universidades

encuestadas por países (continuación)

\begin{tabular}{lc}
\hline \multicolumn{1}{c}{ País } & Porcentaje \\
\hline México & 18 \\
\hline Nicaragua & 2 \\
\hline Panamá & 3 \\
\hline Paraguay & 2 \\
\hline Perú & 12 \\
\hline Puerto Rico & 1 \\
\hline Rep. Dominicana & 1 \\
\hline Venezuela & 5 \\
\hline
\end{tabular}

La Tabla 2 presenta, complementariamente, la distribución de la muestra en función de la titularidad de las universidades encuestadas.

Tabla 2. Composición de la muestra de universidades encuestadas según su titularidad

\begin{tabular}{lc}
\hline \multicolumn{1}{c}{ Titularidad } & Porcentaje \\
\hline Privada & 28 \\
\hline Privada sin ánimo de lucro & 12 \\
\hline Pública & 59 \\
\hline
\end{tabular}

Adicionalmente, aunque de forma separada a la administración de la encuesta, se realizó un llamado abierto a través del sitio web del Instituto Internacional de la UNESCO para la Educación Superior en América Latina y el Caribe (www.iesalc.unesco.org) para que estudiantes y profesores pudieran compartir su experiencia durante la pandemia. En la web se les ofrecía la oportunidad de publicar un corto video o relato escrito en el que describieran los retos a los que se enfrentaron al enseñar y aprender de forma remota. Estos videos fueron analizados y las principales conclusiones incorporadas a esta misma contribución. En total se recopilaron 37 testimonios (33 mediante videos y 4 relatos escritos); sus protagonistas son procedentes de 4 países de la región (Brasil, Colombia, El Salvador y Perú), estudiantes o profesores de 11 universidades, de las cuales 67 por ciento son de titularidad pública y 33 por ciento privada.

\section{RESUlTADOS}

La mayoría de las universidades contaban ya con más de una plataforma apta para la enseñanza virtual desde antes de la pandemia. Las universidades están intentando garantizar la continuidad pedagógica adoptando soluciones que faciliten una enseñanza remota de emergencia y, progresivamente, a medida que la duración de la crisis se alarga, hacerlas evolucionar hacia una enseñanza virtual más cualificada, estabilizando las herramientas y mejorando las capacidades de los docentes. En el caso de la educación superior estas soluciones se han fundamentado, en primer lugar, en algún tipo de plataforma tecnológica en la que se pueden ofrecen clases virtuales, se publican materiales didácticos y se garantiza la comunicación pedagógica, tanto con el respectivo docente como con el resto de los estudiantes. En la región, la gran mayoría de las universidades (80 por ciento) contaba ya con una plataforma tecnológica apta para la educación a distancia desde antes de la pandemia

\section{Gráfico 1. Existencia previa de plataformas tecnológicas para la educación a distancia}

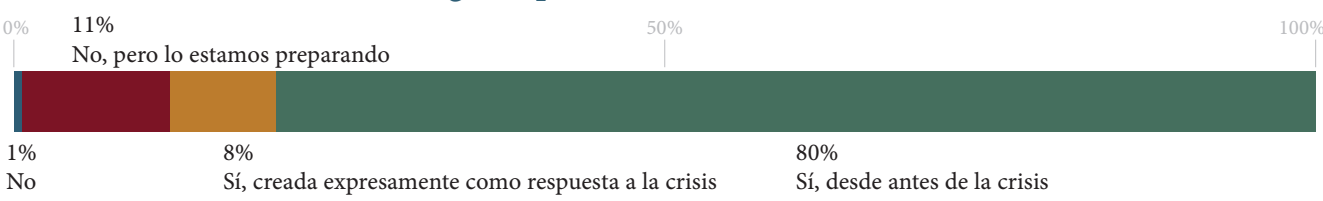


y un 8 por ciento adicional la creó o puso en funcionamiento como respuesta inmediata a la crisis, tal y como muestra el Gráfico 1.

Apenas hay diferencias entre las universidades de acuerdo con su titularidad, aunque las privadas sin ánimo de lucro son las que en mayor porcentaje contaban ya con una plataforma, mientras que sólo tres cuartas partes de las públicas la tenían. Los países con una situación de partida más difícil en este sentido son, y por este orden, Venezuela, Chile, Ecuador y Perú con sólo entre un 49 por ciento y un máximo de un 70 por ciento de universidades previamente equipadas con una plataforma tecnológica capaz de soportar la educación remota de emergencia.

En la región, la plataforma más adoptada por las universidades es Moodle (60 por ciento), con cierta distancia respecto de las restantes como Google Classroom (30 por ciento) y Blackboard (7 por ciento) y multitud de otras comerciales, por una parte, y plataformas de diseño y producción propia de las mismas universidades ( 21 por ciento), por otra. En realidad, lo que estas cifras muestran es una cierta división de opciones entre partidarios de soluciones abiertas, comerciales o de propia concepción; cada una de estas alternativas tiene sus ventajas e inconvenientes, al mismo tiempo que son indicativas de culturas organizativas distintas.

Pero debe hacerse constar igualmente un fenómeno muy frecuente: que dentro de una misma institución conviven plataformas distintas, cosa que sucede en un 80 por ciento de los casos. Se trata de una cuestión compleja en la que confluyen varios factores. Por una parte, algunas de las plataformas en realidad no son redundantes; este es el caso de Microsoft Teams, que se usa en un 11 por ciento de instituciones fundamentalmente para videolecciones o seminarios sincrónicos con video completando las capacidades de otras plataformas como Moodle; de hecho, sólo en la mitad de esos casos Microsoft Teams es la única plataforma disponible. Menor aún es la penetración de Zoom, que sólo alcanza a un 4 por ciento de las universidades, una aplicación que ninguna universidad usa de forma aislada como único medio de comunicación. Además, aunque las plataformas que ofrecen video sincrónico de calidad pueden ser muy interesantes y útiles, lo cierto es que también exigen contar con un buen equipamiento $y$ banda ancha que, como se verá más adelante, no es la situación mayoritaria. Por otra parte, la proverbial autonomía de las facultades y los departamentos, particularmente en establecimientos públicos, posibilita que dentro de una misma universidad se opte por soluciones distintas denotando falta de coordinación y, lo que aún es peor, la pérdida de oportunidades de economías de escala.

La radio y la televisión también se están usando para la educación superior a distancia. Décadas atrás, universidades y, en algunos casos, países enteros contaban con emisiones de televisión y de radio educativa que, con la llegada de Internet, han ido desapareciendo progresivamente o se han quedado en una posición relativamente marginal. La decadencia de estos medios de enseñanza en la educación superior ha impedido que emergieran durante la crisis como una alternativa mayoritaria para la transmisión de contenidos. Pero, aun así, se están utilizando todavía en alrededor de un tercio de los casos; más concretamente, un 18 por ciento de las universidades cuenta con ambos medios, un 8 por ciento sólo con radio y un 3 por ciento sólo con televisión. Sin embargo, estos medios siempre se usan simultáneamente con plataformas digitales que, en última instancia, se han configurado como el soporte fundamental para la enseñanza superior de emergencia.

El uso real de las plataformas está lejos de ser universal: sólo en una cuarta parte de las universidades, 100 por ciento del profesorado y de los estudiantes la usan con regularidad. Más allá de la opción tecnológica utilizada, la cuestión de fondo es cuál es el uso real de las plataformas tanto por parte del profesorado 
como por parte de los estudiantes y, por tanto, cuál es su alcance. En este sentido, parece claro que existe una gran variabilidad en la región y que sólo en contadas excepciones se puede hablar de un alcance prácticamente universal. Las cifras, en promedio, muestran un nivel de uso que se podría calificar de mayoritario como muestra el Gráfico 2: el 68 por ciento del profesorado se conecta con regularidad a su correspondiente plataforma y en el caso de los estudiantes ese porcentaje se eleva hasta un 80 por ciento. Los países donde las tasas de uso son más elevadas son Colombia, México, Argentina y Perú, por este orden, y donde son más bajas es en Brasil, la República Dominicana y Bolivia.

Gráfico 2. Porcentaje universidades según el volumen de docentes y estudiantes conectados, en tramos de 25 puntos

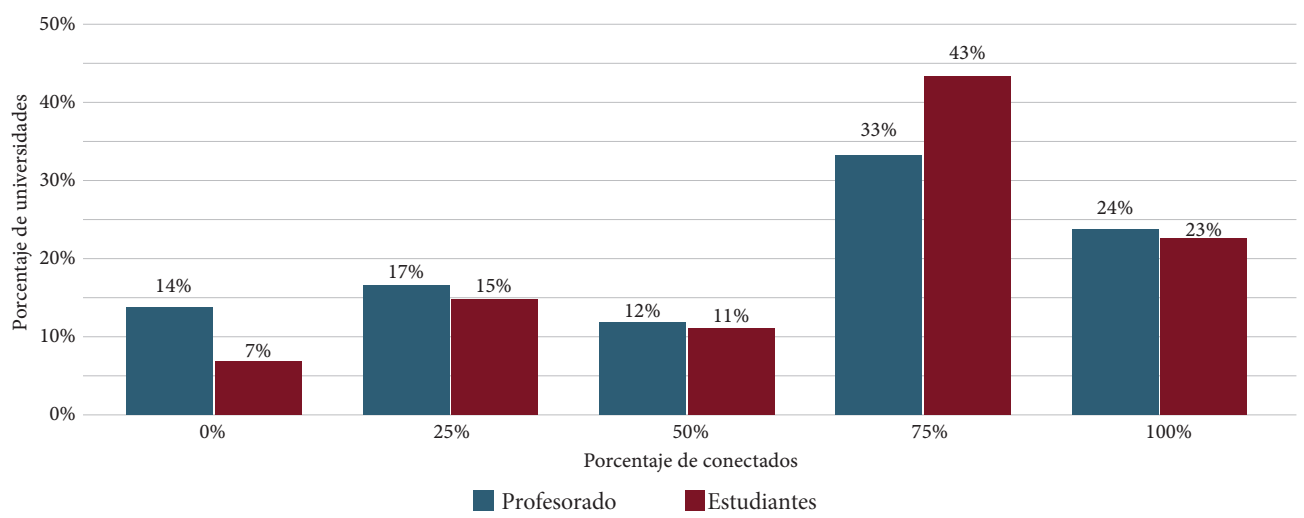

En casi la mitad de las universidades más del 50 por ciento del profesorado usa la plataforma con regularidad y en apenas una cuarta parte de las universidades se afirma que el porcentaje asciende hasta el 100 por ciento del profesorado. En tan sólo un 14 por ciento de universidades se afirma que el porcentaje de docentes usuarios de la plataforma es nulo. $\mathrm{Si}$, complementariamente, se examinan los resultados del alcance del uso de las plataformas por parte de los estudiantes, la imagen arroja que en un 66 por ciento de las universidades más de la mitad de los estudiantes se conecta con regularidad y en el 23\% de ellas se afirma que el porcentaje es equivalente al 100 por ciento. En el otro extremo, sólo en un 7 por ciento de los casos, los estudiantes no se conectan nunca. En definitiva, pareciera que el alcance de las plataformas es algo mayor en el caso de los estudiantes que en el del profesorado.
Las principales dificultades para el aprovechamiento de las plataformas son la falta de competencias digitales de los docentes, incluso por encima de la falta de conectividad apropiada en los hogares. Las universidades mencionan distintos tipos de dificultades para explicar la falta de un alcance universal. En primer lugar, se hace referencia a la falta de competencias digitales de los docentes (65 por ciento) e incluso de los estudiantes (49 por ciento) y en un porcentaje importante de unos y de otros simultáneamente (39 por ciento). He ahí, pues, un problema que las universidades tienen dificultad para resolver porque, en las actuales circunstancias, no les queda más remedio que utilizar precisamente la misma plataforma para desarrollar esas competencias. En segundo lugar, está la cuestión del limitado acceso a Internet en los hogares (58 por ciento) pero cuya magnitud es percibida como menor frente a las dificultades 
generadas por la falta de competencias. En tercer lugar, está la cuestión de la capacidad de los servidores de la universidad para soportar el tráfico generado durante la emergencia (32 por ciento) que, a todas luces, parece haber desbordado las posibilidades existentes. Las universidades reconocen, finalmente, que el propio diseño de las plataformas y su configuración pueden generar problemas (22 por ciento) que dificulten su uso y que, en última instancia, provoquen situaciones de desafección y, desgraciadamente, incluso de abandono.

Para favorecer la continuidad pedagógica las universidades no proponen una única metodología: mayoritariamente recomiendan al profesorado el uso de su correspondiente aula virtual, pero también se fomenta la impartición de videolecciones. Tanto o más importante que la existencia de las plataformas es la forma en que se utilizan para garantizar la continuidad pedagógica. Es obvio que no todos los docentes contaban desde antes de la pandemia ni con experiencia previa en el manejo de las plataformas ni, lo que aun importa más, con una formación específica para la educación superior a distancia; de hecho, en muchos países en todo el mundo, la formación pedagógica docente está lejos de ser la norma en la educación superior. La confrontación del docente con la realidad de la plataforma o de cualquier otro soporte con el que debe ahora garantizar la continuidad pedagógica se ha dado en un contexto de emergencia que no ha permitido contrarrestar a tiempo este déficit inicial de capacitación y experiencia. Los cursos se han reanudado, o iniciado, con la mejor voluntad, pero no siempre con las competencias deseables. Además, el respeto al principio de la libertad de cátedra o, más en general, a la autonomía de cada docente ha frenado el avance de propuestas de estandarización metodológica o de la creación de protocolos comunes, con todas las variaciones necesarias en atención a la diversa naturaleza de los estudiantes y de los objetivos perseguidos. En muchos casos, esta limitación se ha saldado con déficits pedagógicos importantes; en otros, las características de la solución tecnológica adoptada han terminado imponiendo las condiciones del ejercicio docente limitándolas, por ejemplo, a la posibilidad de impartir videoconferencias.

La disponibilidad de las plataformas permite explicar el por qué en la mayoría de las universidades la propuesta metodológica que se recomienda al profesorado es el uso de la correspondiente aula virtual, a través de la cual pueden publicar contenidos, proponer actividades didácticas, evaluar y, por supuesto, comunicarse con los estudiantes. Alrededor de un 78 por ciento de las universidades promueven esta aproximación. La segunda opción preferida por las universidades consiste en el recurso a las clases magistrales emitidas como videos, sincrónica o asincrónicamente, cosa que sucede en el 41 por ciento de las universidades, como muestra el Gráfico 3.

\section{Gráfico 3. Opciones metodológicas recomendadas Las opciones no son excluyentes}

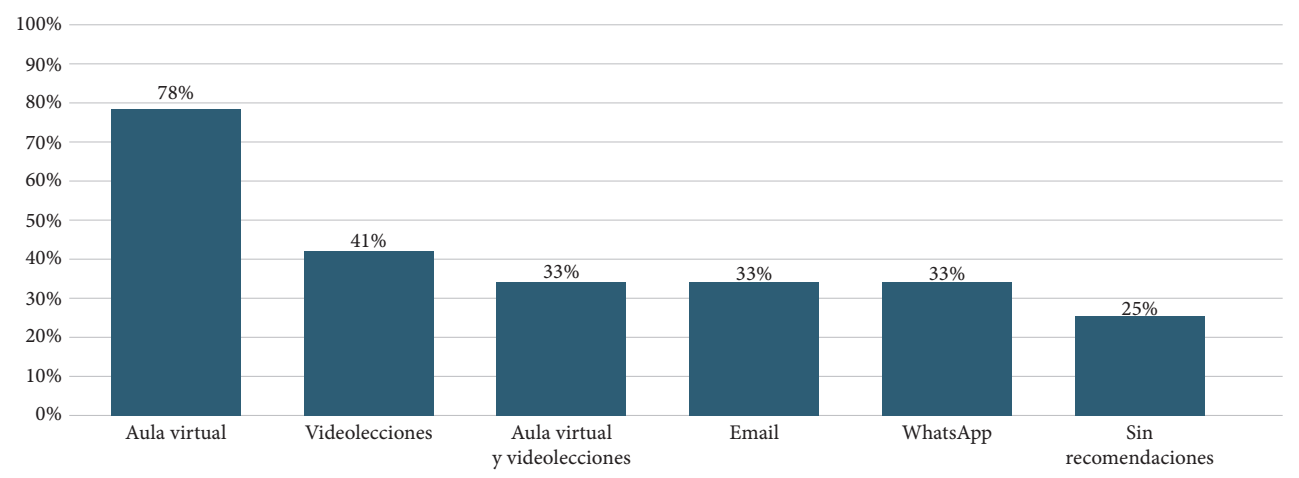


En un tercio de las universidades se opta por promover ambas cosas, las aulas virtuales y las videoconferencias, simultáneamente. También en aproximadamente otro tercio de las universidades, donde las plataformas son inexistentes o de bajo alcance, lo que promueven es el recurso al correo electrónico o a WhatsApp como mecanismo de comunicación y seguimiento de los estudiantes o, definitivamente, sugieren recurrir a metodologías que reduzcan al mínimo la necesidad de una conectividad constante. Finalmente, en una quinta parte de las universidades se opta por dejar a criterio de cada docente, con total libertad, la aproximación metodológica que desee poner en práctica, con o sin uso de la tecnología.

Los estudiantes comparten las mismas situaciones problemáticas. El análisis de los testimonios de los estudiantes revela que existen, fundamentalmente, cuatro órdenes de preocupación: acceso tecnológico, dificultades económicas, aislamiento social, e inconvenientes pedagógicos. En general, los estudiantes que han tenido que dejar las grandes ciudades en las que se ubican sus universidades para volver al interior de sus países son los más proclives a documentar problemáticas de acceso, ya sea por falta de tecnología o de conectividad. También en algunos casos se hace referencia a la falta de competencias digitales, pero éstas las echan más en falta cuando se refieren al uso que el profesorado hace de las plataformas. En segundo lugar, también es frecuente que se cite la problemática financiera derivada de la baja actividad económica que pueden llevar a cabo los estudiantes en el contexto del confinamiento total o parcial. También, desde el primer momento parece claro que los estudiantes echan en falta los componentes de relación social que giran alrededor de la experiencia universitaria; muchos anticipan ya el impacto que el aislamiento social podría llegar a tener sobre su estado emocional. Y, finalmente, reportan elementos pedagógicos que los estudiantes describen como falta de capacidades pedagógicas del profesorado que les impide ajustar su actividad docente a las limitaciones, exigencias y también oportunidades que ofrece la educación remota, en particular aquéllas con soporte tecnológico. En algunos casos, los estudiantes también mencionan la necesidad de generar hábitos y rutinas que les permitan gestionar apropiadamente, de forma autónoma, su propio proceso de aprendizaje, aspecto al que, al parecer, no están suficientemente habituados en el contexto de un sistema pedagógico que no les confiere la autonomía necesaria.

Las universidades han desplegado estrategias de apoyo, fundamentalmente a los estudiantes, en los frentes tecnológico, pedagógico y socioemocional, pero no en el financiero. Son muy notables los esfuerzos que las universidades han venido realizando para ofrecer soporte a la comunidad universitaria con el objetivo de garantizar la continuidad pedagógica en las mejores condiciones. Estos apoyos, generalmente, cubren tres frentes: el tecnológico, con el objetivo de hacer llegar conectividad o equipamiento a quien carezca de ellos; el pedagógico, destinado a desarrollar las competencias básicas para facilitar el aprovechamiento de las posibilidades de la educación a distancia; y, finalmente, el socioemocional, que busca reducir la ansiedad y el estrés que el aislamiento y la desconexión social pueden generar. Es importante destacar que las universidades consideran que estas iniciativas son verdaderas innovaciones. De hecho, cuando se les pregunta acerca de las innovaciones desarrolladas para hacer frente a la pandemia, existe una enorme coincidencia en estos tres ámbitos. Propiamente hablando, no se trataría de innovaciones, es decir, de formas nuevas de actuación para resolver situaciones o cambiar procesos; más bien, se trata de iniciativas de las que antes carecían las universidades y de ahí que ellas mismas las consideren innovaciones, pues no tenían referencias previas.

Aunque estos tres frentes podrían cubrir por igual a todos los miembros de la comunidad universitaria, lo cierto es que la prioridad sigue siendo, por razones comprensibles, 
favorecer a los estudiantes, en particular a los más vulnerables. Por supuesto, existe un cuarto frente, el de las ayudas financieras, que, en la realidad, se ha mostrado prácticamente inexistente porque sólo unas pocas universidades de la región cuentan con la capacidad financiera requerida para ofrecer ayudas económicas extraordinarias durante la pandemia.
Los resultados obtenidos, como muestra el Gráfico 4, reflejan con toda claridad que un porcentaje importante de las universidades (44 por ciento) ofrece a todos sus estudiantes simultáneamente apoyos tecnológicos, pedagógicos y socioemocionales, siendo estos dos últimos los más frecuentes ( 62 por ciento en ambos casos).

\section{Gráfico 4. Apoyos ofrecidos a los estudiantes \\ Las opciones no son excluyentes}

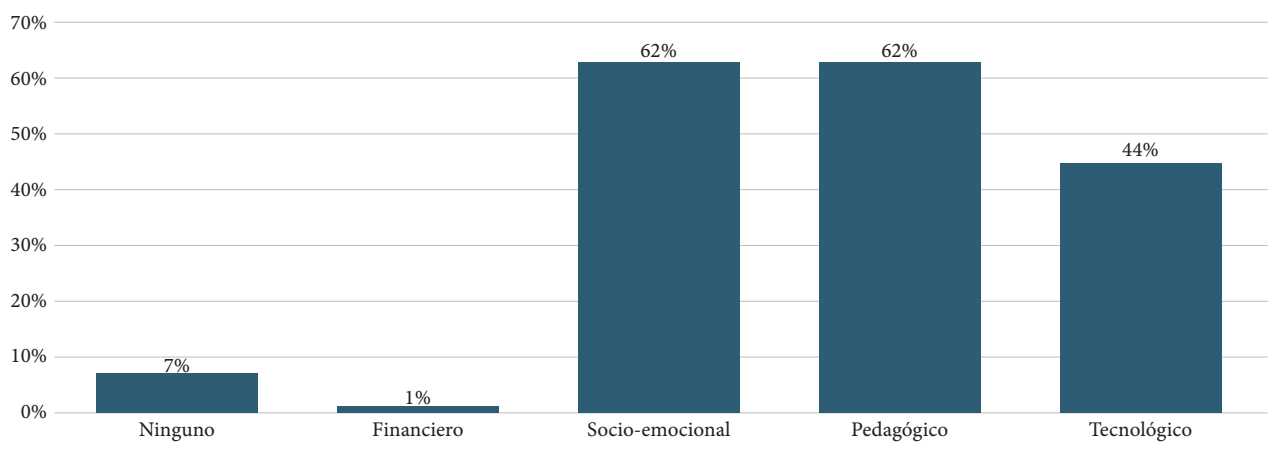

Sólo un porcentaje relativamente bajo no ofrece ninguno de estos tipos de apoyo (7 por ciento), pero aún es menor (1 por ciento) el número de universidades que tiene la capacidad de ofrecer algún tipo de apoyo financiero directo a sus estudiantes, una capacidad que en la mayor parte de los países está sólo al alcance de las administraciones públicas o de las entidades, públicas o privadas, en la modalidad de crédito educativo.

La mitad de las universidades han sido consultadas por sus respectivos gobiernos para formular sus estrategias durante la crisis. Pero la valoración que hacen de las políticas diseñadas no es muy positiva y aún lo es menos cuando se trata de los planes para después de la crisis. La continuidad pedagógica que están garantizando las universidades tiene lugar en un particular entorno en el que los gobiernos (nacionales, estatales, municipales) pueden generar mejores condiciones de operación. Un ejemplo claro lo constituyen los cambios normativos que, en multitud de países, han dado carta de naturaleza a la educación superior a distancia para las titulaciones de grado o han hecho posible que, aunque no hubieran sido previamente acreditadas en la modalidad virtual, puedan impartirse provisionalmente bajo ella hasta que la pandemia permita reabrir las aulas. Desde este punto de vista, es importante que las universidades sean consultadas, que tomen parte en la conversación acerca de cómo generar el entorno más propicio para la continuidad pedagógica y que conozcan los planes sanitarios que se están diseñando para la vuelta a las aulas y que participen de su diseño. Obviamente, muchas de estas consultas se realizan indirectamente a través de los consejos de universidades, y sus equivalentes, y de las redes de universidades, pero en esta situación de emergencia la consulta y el conocimiento directo de la realidad de cada institución parecen más necesarios que nunca.

Aproximadamente la mitad de las universidades (52 por ciento) declaran haber sido 
consultadas directamente por sus respectivos gobiernos con respecto a las medidas a tomar para garantizar la continuidad pedagógica, lo cual es una cifra realmente elevada y que, probablemente, se explique por el carácter inédito, sin precedentes, de la crisis experimentada y por la necesidad de forjar consensos nacionales de largo alcance. Visto el número de instituciones que existen en la región, que la mitad haya sido consultada de algún modo sobre cómo encarar la crisis es un dato que sólo admite una lectura extremadamente positiva y dice mucho acerca de cómo buena parte de los gobiernos en la región formulan sus políticas y estrategias sectoriales en educación superior, intentando forjar consensos. Sólo en Bolivia y Guatemala no parecen haberse producido este tipo de consultas.

Otra cosa bien distinta es si las propuestas finalmente lanzadas por los respectivos gobiernos para salvaguardar la calidad y la equidad en la educación superior durante la emergencia han sido bien recibidas por las universidades. En materia de estrategias para preservar la calidad, los gobiernos de la región reciben una nota media de 2.5 sobre 5 , es decir, un aprobado justo. En materia de equidad la nota es más baja, apenas de 2.3 sobre 5 que, sin ser un resultado desastroso, es obviamente menos satisfactorio. Ligeramente más preocupante es cómo enjuician las universidades los planes de sus respectivos gobiernos en relación con la educación superior para después de la pandemia, con una puntuación de solo 2.2 sobre 5. En cierto modo, no es extraño que así sea porque las incertidumbres acerca de cuándo y cómo se volverán a abrir las universidades y, aún más, acerca de la prioridad que se otorgue al sector en los futuros presupuestos públicos, contribuyen a explicar por qué esos planes gubernamentales reciben una valoración baja.

Los países donde los planes de futuro son mejor valorados por las universidades son, por este orden, Perú, Chile, Colombia y República Dominicana, mientras que aquellos que merecen puntuaciones más bajas son Guatemala,
Bolivia y El Salvador. Es interesante destacar igualmente que en Nicaragua y Venezuela las opiniones de las universidades están fuertemente polarizadas entre quienes dan buena y mala nota a esos planes.

\section{DISCUSIÓN Y CONCLUSIONES: ENCARANDO EL DÍA DESPUÉS}

Aunque la incertidumbre todavía planea sobre el horizonte, parece claro que la reapertura no significará la vuelta a la normalidad docente e investigadora tal y como la conocimos, ni tampoco será abrupta, como lo fue la clausura (Blofield et al., 2020). Partiendo del ejemplo de lo que ya está sucediendo con la reapertura de las escuelas e instituciones de educación superior de diferentes países tanto en Asia como en Europa, parece plausible que la reapertura se hará con estrictas medidas sanitarias que se traducirán en: a) grupos de estudiantes más reducidos en las aulas cuyo volumen dependerá de las condiciones espaciales de las aulas y de las instituciones; y b) con un menor número de clases presenciales por grupo, por imperativo de la disponibilidad de espacios. En definitiva, lo más probable es que las formas de enseñanza y aprendizaje que han empezado como fórmulas de emergencia para garantizar la continuidad pedagógica evolucionen y se consoliden ya desde la reapertura como parte del modelo híbrido con el que habrá que convivir de momento y que tal vez se convierta en la nueva normalidad pedagógica en la educación superior en el contexto de una previsible reestructuración de la provisión.

Hay dos estrategias fundamentales para encarar esta previsible, y deseable, reestructuración. La primera es recuperar y la segunda es rediseñar. De hecho, no habría que esperar a la reapertura para empezar a desplegar estas estrategias, sino que las instituciones deberían asumirlas ya como parte de su compromiso con el futuro.

Recuperar implica diseñar medidas pedagógicas para evaluar formativamente y generar 
mecanismos compensatorios de apoyo al aprendizaje, en particular de los estudiantes en desventaja. La tecnología puede ser utilizada como una herramienta de apoyo para la personalización de las actividades de nivelación. Aunque existen herramientas tecnológicas muy sólidas de evaluación, parece más recomendable, y fácil de gestionar, modificar los instrumentos para favorecer una evaluación más abierta y asincrónica.

En este sentido, hay algunas estrategias que, a pesar de ser poco frecuentes en el ámbito de la educación superior, pudieran dar buenos frutos como, por ejemplo:

- tutorización individualizada;

- grupos reducidos de aprendizaje para la nivelación en materias críticas por su carácter instrumental; $y$

- escuelas de verano (o de invierno) que ofrezcan seminarios compensatorios. Inevitablemente, la puesta en marcha de iniciativas como éstas conlleva un coste asociado nada despreciable, pero los beneficios en términos de calidad de aprendizajes y de equidad, sobrepasan con mucho los costes.

En paralelo, hay que planificar cómo se debe reestructurar la provisión formativa y esto requiere una estrategia de rediseño que debería centrarse en tres grandes ejes (Pedró, 2020a):

1. Documentar los cambios pedagógicos introducidos durante la crisis y sus impactos; en particular, hay que prestar atención a los efectos negativos de la educación a distancia de emergencia y, particularmente, al síndrome del coronateaching. La pregunta crítica es si la experiencia adquirida puede capitalizarse para un rediseño de estos procesos, maximizando las ventajas de las clases presenciales al tiempo que se extrae mayor partido de las tecnologías, y, en segundo lugar, establecer hasta dónde quiere o puede llegar cada institución.

2. Promover la reflexión interna sobre la renovación del modelo de enseñanza y aprendizaje. Esta reflexión podrá llevarse mejor a cabo si las instituciones de educación superior cuentan con oficinas de innovación y apoyo pedagógico cuyo papel, además de desarrollar las competencias pedagógicas del profesorado, es el de fomentar la innovación pedagógica y acumular y diseminar las evidencias que resulten de su evaluación.

3. Aprender de los errores y escalar la digitalización, la hibridación y el aprendizaje ubicuo. Pensando en el futuro hay que partir del principio del realismo y generar estrategias que no confíen sólo en una única tecnología, sino que combinen varias para garantizar llegar a todos los estudiantes o, lo que es tanto o más importante, que las soluciones tecnológicas no perjudican a quienes ya parten de una situación de desventaja. Cada institución, y probablemente cada disciplina, deben encontrar la combinación de tecnologías y recursos más apropiadas para mejorar el impacto pedagógico sin renunciar a la equidad y a la inclusión.

Se acostumbra a decir que en cada crisis hay siempre una oportunidad. Tal vez, en este caso, sea la de la revisión pedagógica y la reestructuración de la oferta formativa en la educación superior. Es de esperar, en este sentido, que sean muchas las instituciones que emprendan el camino de una necesaria renovación pedagógica que favorezca tanto la calidad como también la equidad. 


\section{REFERENCIAS}

Amemado, D. (2020), “COVID-19: un impulsor inesperado e inusual de la educación en línea", Educación Superior Internacional, 102, pp. 12-14, en: https://www.internationalhighereducation.net/api-v1/article/!/action/getPdfOfArticle/articleID/2905/productID/29/ filename/articl e-id-2905.pdf

Blofield, M., B. Hoffmann y M. Llanos (2020), "Evaluación del impacto político y social de la crisis del COVID-19 en América Latina" (GIGA Focus Lateinamerika, 3), GIGA Instituto Alemán de Estudios Globales y de Área-Leibniz-Institut für Globale und Regionale Studien, Institut für Lateinamerika-Studien, en: https://www.gigahamburg.de/en/publications/18749084-assessing-political-social-impact-covid-19-crisisamérica latina/

Camaño Morúa, C., D. Axel, C. Célia, M. Bernardo Sfredo, V. Damián y P. Daniela (2020), Educación superior y Covid-19 en América Latina, en: http://www.guninetwork.org/files/ensayo_axel_et_al._long.pdf

Crawford, J., K. Butler-Henderson, J. Rudolph, B. Malkawi, M. Glowatz, R. Burton, P. Magni y S. Lam, S. (2020), “COVID-19: respuestas pedagógicas digitales intra-período de educación superior de 20 países", Revista de Aprendizaje Aplicado y Enseñanza, vol. 3, núm. 1, pp. 1-20. DOI: https://doi.org/10.37074/jalt.2020.3.1.7

FARNELL,T.,A.SkledarMatijevićyN.ŠćukanecSchmidt (2021), El impacto de COVID-19 en la educación superior: una revisión de la evidencia emergente, Oficina de Publicaciones de la Unión Europea, en: https://op.europa.eu/en/publicationdetail/-/publication/876ce591-87a0-11ebac4c-01aa75ed71a1/language-es

GazcA Herrera, L.A. (2020), "Implicaciones del coronavirus COVID-19 en los procesos de enseñanza en la educación superior", RIDE. Revista Iberoamericana para la Investigación y el Desarrollo Educativo, vol. 11, núm. 21. DOI: https://doi.org/10.23913/ride.v11i21.753
Goedegebuure, L. y L. Meek (2021), “Crisis - ¿Qué crisis?”, Estudios en Educación Superior, vol. 46, núm. 1, pp. 1-4. DOI: https://doi.org/1 0.1080/03075079.2020.1859680

Hershberg, E., A. Flinn-Palcic y C. Kambhu (2020), La pandemia de COVID-19 y las universidades latinoamericanas, Centro de Estudios Latinoamericanos y Latinos (CLALS) de la American University en Washington, en: https://www.american.edu/centers/latinamerican-latino-studies/upload/la-highered-covid-final.pdf

Marinoni, G., H. Van't Land y T. Jensen (2020), El impacto del COVID-19 en la educación superior de todo el mundo. Informe de la Encuesta Global de la IAU, en: https://www.iau- aiu. net/IMG/pdf/iau_covid19_and_he_survey_ report_final_may_2020.pdf

Pedró, F. (2020a), “COVID-19 y educación superior en América Latina y el Caribe: efectos, impactos y recomendaciones políticas", Análisis Carolina, vol. 36, núm. 1, pp. 1-15. DOI: https://doi.org/10.33960/ac_36.2020

Pedró, F. (2020b), "COVID-19 y educación superior: crisis y ventanas de oportunidad”, en I. Dussel, P. Ferrante y D. Pulfer (eds.), Pensar la educación en tiempos de pandemia II. Experiencias y problemáticas en Iberoamérica UNIPE, pp. 73-82. DOI: https://doi.org/10.5965 /1984723822482021303

SAlto, D. (2020), “COVID-19 y la educación superior en América Latina: desafíos y posibilidades en la transición a la educación en línea", eLearn, núm. 9. DOI: https://doi. org/10.1145/3424971.3421751

UNESCO-IESALC (2020), COVID-19 y educación superior: hoy y mañana. Análisis de impacto, respuestas politicas y recomendaciones, UNESCO-IESALC, en: https://www.iesalc. unesco.org/en/wp-content/uploads/2020/04/ COVID-19-EN- 090420-2.pdf 